\title{
The First Measurement of the Longitudinal Diffractive Structure Function $F_{L}^{D}$
}

\author{
David Š́lek ${ }^{1}$, on behalf of H1 Collaboration \\ 1- Institute of Particle and Nuclear Physics, Charles University \\ Prague, Czech Republic
}

\begin{abstract}
The first measurement of the longitudinal diffractive structure function $F_{L}^{D}$ is presented, using the $\mathrm{H} 1$ detector at HERA. The structure function is extracted from the first measurements of the diffractive cross section at centre of mass energies $\sqrt{s}$ of $225 \mathrm{GeV}$ and $252 \mathrm{GeV}$, together with a new measurement at $\sqrt{s}$ of $319 \mathrm{GeV}$, using data taken in 2007 at high values of inelasticity $y$. The measured $F_{L}^{D}$ is compared to predictions from NLO QCD fits to previous measurements of the inclusive diffractive DIS cross section.
\end{abstract}

\section{Introduction}

The diffractive deep-inelastic cross section can be expressed in terms of the kinematic variables $Q^{2}, \beta=\frac{Q^{2}}{Q^{2}+M_{X}^{2}}$ and $x_{\mathbb{P}}=\frac{Q^{2}+M_{X}^{2}}{Q^{2}+W^{2}}$, where $M_{X}$ is the mass of the diffractive system and $W$ is the invariant mass of the gamma-proton system. The cross section formula then reads

$$
\frac{d^{3} \sigma^{e p \rightarrow e X Y}}{d x_{\mathbb{P}} d \beta d Q^{2}}=\frac{2 \pi \alpha^{2}}{\beta Q^{4}} Y_{+} \sigma_{r}^{D}\left(x_{\mathbb{P}}, \beta, Q^{2}\right),
$$

where $\sigma_{r}^{D}$ is the diffractive reduced cross section. Similarly to the description of inclusive $e p$ scattering, it consists of two structure functions, $F_{2}^{D}$ and $F_{L}^{D}$, where

$$
\sigma_{r}^{D}=F_{2}^{D}-\frac{y^{2}}{Y_{+}} F_{L}^{D}
$$

The longitudinal structure function $F_{L}^{D}$ is scaled by a kinematic factor $\frac{y^{2}}{Y_{+}}$, where $Y_{+}=1+(1-y)^{2}$. This kinematic factor is non-negligible only at high values of the inelasticity $y$. Therefore, the term containing the longitudinal structure function is often neglected in cross section measurements.

The diffractive proton structure function $F_{2}^{D}$ constrains quark parton densities. Without knowledge of $F_{L}^{D}$, gluon densities are only constrained weakly from the scaling violations. Since $F_{L}^{D} \sim x g(x)$ is directly sensitive to the gluons, the measurement of $F_{L}^{D}$ provides a crucial test of the concept of parton densities and QCD factorisation in diffraction.

Models, such as BEKW [2], predict higher twist longitudinal contributions to the diffractive structure function $F_{2}=F_{T}+F_{L}$ at high $\beta$ and low $Q^{2}$. In this sense, the measurement of $F_{L}^{D}$ gives insight into an as yet unexplored area of diffraction.

\section{Measurement Strategy}

The $F_{L}^{D}$ analysis adopts the basic measurement strategy from the $F_{L}$ analysis [3, 4]. Similarly to that analysis, the two structure functions in the reduced cross section $\sigma_{r}^{D}=F_{2}^{D}-\frac{y^{2}}{Y_{+}} F_{L}^{D}$ 
can be separated only if at least two cross section measurements at fixed $Q^{2}, \beta, x_{\mathbb{P}}$ and different values of inelasticity $y$ are available. Since the kinematic variables are bound by $Q^{2}=x_{\mathbb{P}} \beta y s$ the only way to vary $y$ while keeping the other variables fixed is to measure the cross section at different centre of mass energies $\sqrt{s}$. The kinematic factor $\frac{y^{2}}{Y_{+}}$in Eq. (2) suppresses the contribution of $F_{L}^{D}$ at low $y$. Therefore, high $y$ data is crucial for the $F_{L}^{D}$ extraction.

The last months of HERA data taking were devoted to runs with reduced proton beam energy. Prospects for the measurement of $F_{L}^{D}$ and related Monte Carlo studies [5] played an important role in planning the amount of reduced proton beam energy data to be taken. HERA collided 27.6 GeV positrons with protons of different energy. There were $13 \mathrm{pb}^{-1}$ taken at the proton energy $E_{p}=460 \mathrm{GeV}$ and $6 \mathrm{pb}^{-1}$ taken at $E_{p}=575 \mathrm{GeV}$. This data, together with the final data taken at the nominal proton beam energy $E_{p}=920 \mathrm{GeV}$, make it possible to measure the reduced cross section $\sigma_{r}^{D}\left(Q^{2}, \beta, x_{\mathbb{P}}\right)$ for three different values of $y$. Such cross sections can than be plotted in Rosenbluth plots as a function of $\frac{y^{2}}{Y_{+}}$, where the separate proton structure functions can be easily read as $\sigma_{r}^{D}\left(\frac{y^{2}}{Y_{+}}=0\right)=F_{2}^{D}$ and $\sigma_{r}^{D}\left(\frac{y^{2}}{Y_{+}}=1\right)=F_{2}^{D}-F_{L}^{D}$.

Diffractive events for the analysis are selected using the rapidity gap method. In diffraction, the hadronic final state system is separated from the scattered proton by a gap that can be observed as a lack of response in the forward parts of the $\mathrm{H} 1$ detector. The accuracy of the large rapidity gap method strongly depends on our understanding of the detector noise levels. The forward detectors suffered from high noise at the beginning of reduced proton energy running, making it impossible to use the large rapidity gap method. These runs are not considered in the analysis. In terms of luminosity, $21 \mathrm{pb}^{-1}$, $11 \mathrm{pb}^{-1}$ and $6 \mathrm{pb}^{-1}$ of data at centre of mass energies $319 \mathrm{GeV}, 225 \mathrm{GeV}$ and $252 \mathrm{GeV}$, respectively, are used.

Data is analysed in the kinematic region of $Q^{2}>7 \mathrm{GeV}^{2}$ and $y<0.9$. Kinematic variables are reconstructed from the scattered positron properties as $y=1-\frac{E_{e}^{\prime}}{E_{e}} \sin ^{2} \frac{\theta_{e}}{2}$ and $Q^{2}=4 E_{e} E_{e}^{\prime} \cos ^{2} \frac{\theta_{e}}{2}$, where $E_{e}$ is the positron beam energy, $E_{e}^{\prime}$ is the energy of the scattered positron and $\theta_{e}$ is its scattering angle. Accessing the high $y$ region means measuring at low $E_{e}^{\prime}$. The scattered positron candidate is detected down to energies of $3.4 \mathrm{GeV}$ with the Spacal calorimeter, while the charge of the candidate is measured using the central tracker and backward silicon tracker. 
Cross section measurements at high $y$, where the largest contribution from $F_{L}^{D}$ is expected, is rather challenging at $\mathrm{H} 1$ due to the presence of photoproduction ${ }^{\text {a }}$ background. In these processes, the positron is scattered at very small angles so that it exits the $\mathrm{H} 1$ detector undetected through the beam pipe. If one of the hadronic final state particles (a pion) is mis-identified as the scattered positron, then the event appears to be a signal DIS event.

Precise $F_{L}$ and $F_{L}^{D}$ measurements crucially depend on an excellent understanding of the photoproduction background. The background contains similar numbers of events with positive and negative charged hadron mis-identified as the scattered positron. The photoproduction background charge asymmetry asym $=N^{+} / N^{-}$ is close to 1 and is known from an independent analysis that analysed photoproduction events directly using dedicated detectors close to the beam pipe. Since the analysis relies on charge reconstruction it is more convenient to talk about wrong charge background rather than photoproduction background. The number of signal events in the $F_{L}^{D}$ analysis can then be evaluated as

$$
N_{\text {signal }}=N^{+}-\operatorname{asym} N^{-} .
$$

The charge mis-reconstruction probability is larger for higher energies of the scattered positron since its curvature in the detector's magnetic field is smaller. Therefore, the wrong charge background is subtracted only up to energies of $E_{e}^{\prime} \sim 17 \mathrm{GeV}$ beyond

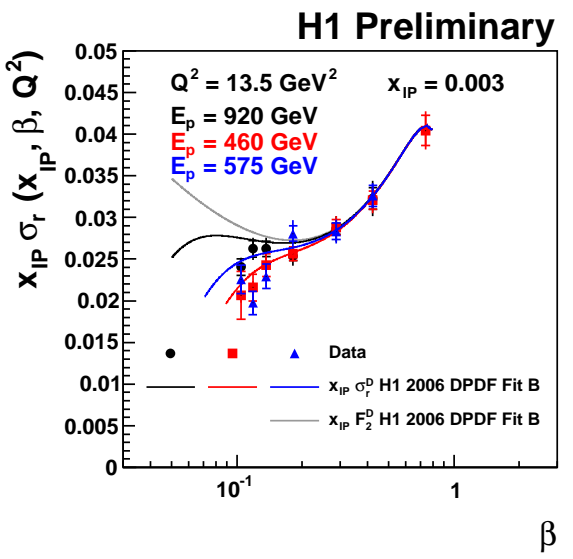

Figure 2: Measurement of the reduced cross section at $E_{p}=460,575$ and $920 \mathrm{GeV}$. The data are compared to an NLO QCD fit to previous H1 data, H1 2006 DPDF Fit B. Also shown is the prediction for $F_{2}^{D}$ from the same fit. The inner error bars are statistical, outer error bars show the statistical and systematic errors added in quadrature.

which photoproduction is not an issue. Figure 1 illustrates the amount of wrong charge background in the $E_{p}=460 \mathrm{GeV}$ data sample.

Figure 2 shows the measured diffractive cross section for all three proton beam energies at $7 \mathrm{GeV}^{2}<Q^{2}<32 \mathrm{GeV}^{2}$ and $0.001<x_{\mathbb{P}}<0.01$ corrected to chosen central values of $Q^{2}=13.5 \mathrm{GeV}^{2}$ and $x_{\mathbb{P}}=0.003$. The cross sections are normalised at $0.28<\beta<0.42$ (low $y$ ) where there are measurements in two bins for all three proton beam energies available. The contribution from $F_{L}^{D}$ is negligible in this $\beta$ range, the suppression factor $\frac{y^{2}}{Y_{+}}$in the lower $\beta$ bin is $1.5 \%$ and $6.9 \%$ for centre of mass energies $319 \mathrm{GeV}$ and $225 \mathrm{GeV}$, respectively. All the measured cross sections are normalised to the analytical prediction for $F_{2}^{D}$ obtained from the H1 2006 DPDF Fit B [6]. The normalisation uncertainty is taken as being correlated between datasets. The figure also shows the analytical predictions for $\sigma_{r}^{D}$ to illustrate the effect of the longitudinal structure function. The reduced cross sections drop at low $\beta$ depending on the factor $\frac{y^{2}}{Y_{+}}$which is different for the three centre of mass energies.

The reduced cross sections measured at fixed $Q^{2}, \beta, x_{\mathbb{P}}$ and different centre of mass energies are then fitted in Rosenbluth plots in order to obtain the longitudinal structure

\footnotetext{
${ }^{\text {a }}$ Processes where the virtuality of the exchanged photon is close to 0 .
} 
function $F_{L}^{D}$. The fits performed for the four lowest $\beta$ cross section points are depicted in Fig. 3.
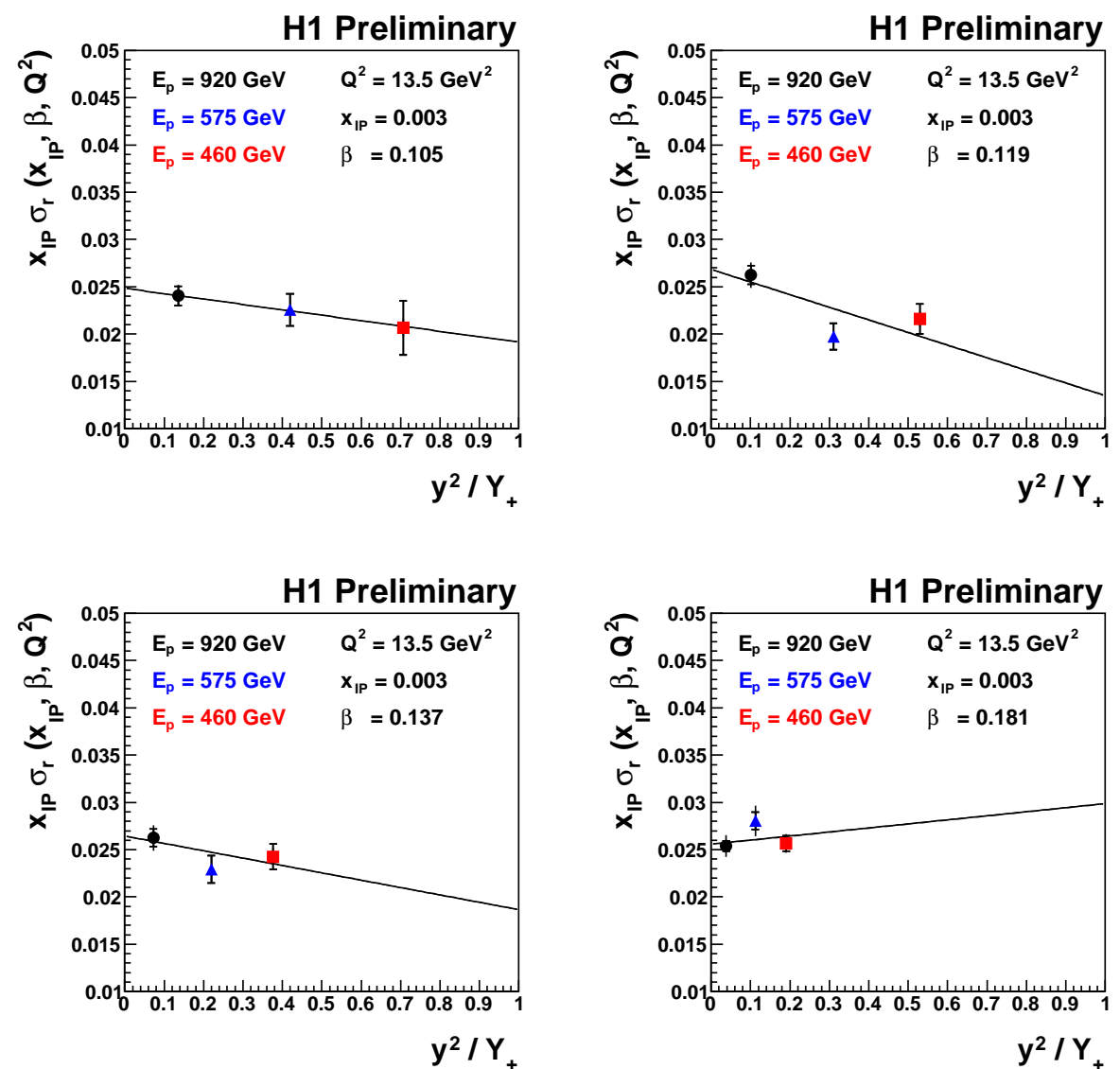

Figure 3: Rosenbluth plots. The reduced cross sections measured at different centre of mass energies are fitted to separate the structure functions $F_{2}^{D}$ and $F_{L}^{D}$.

\section{Results}

The longitudinal diffractive structure function $F_{L}^{D}$ is measured at $7 \mathrm{GeV}^{2}<Q^{2}<32 \mathrm{GeV}^{2}$ and $0.001<x_{\mathbb{P}}<0.01$ for four values of $\beta$. The results are shown in Fig. 4 and represent a measurement of at least $3 \sigma$ significance. The measurement is compatible with the H1 2006 DPDF Fits that are based on diffractive parton densities and QCD factorisation. The results are also compatible with models involving higher twist longitudinal contributions, such as BEKW. However, the analysed $\beta$ region is not sensitive to the higher twist effects (see Fig 4 (right)). 

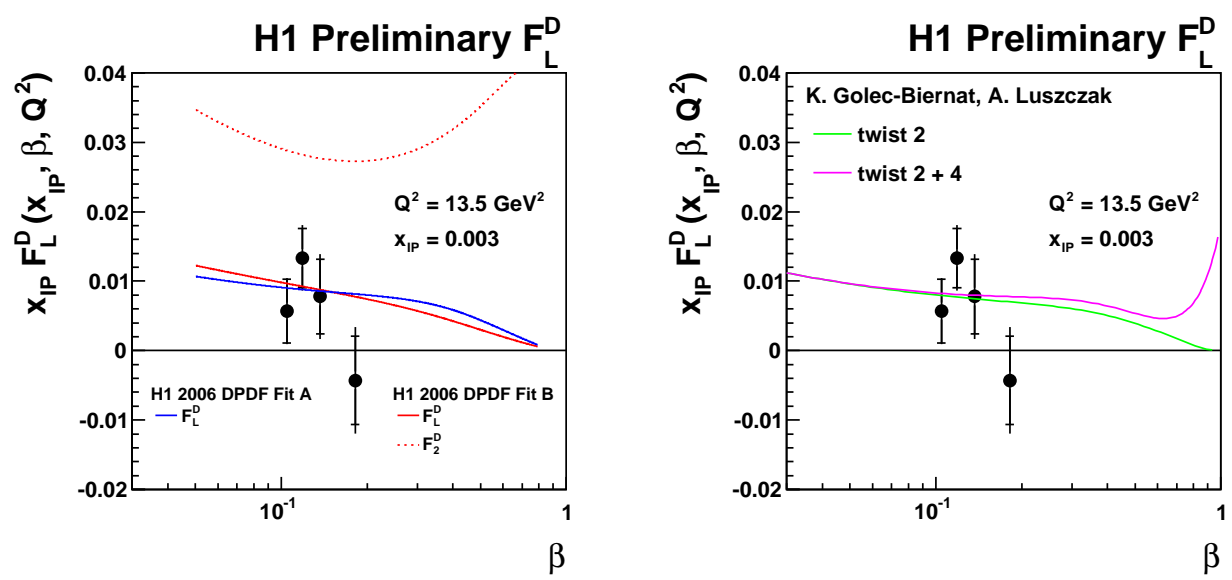

Figure 4: $F_{L}^{D}$ results. (left) Measured $F_{L}^{D}$ compared to H1 2006 DPDF Fits. The dotted line represents the structure function $F_{2}^{D}$ from the Fit B. (right) Measured $F_{L}^{D}$ compared to higher twist predictions. Data do not access high $\beta$ where the predictions differ.

\section{Summary}

The measurement of the longitudinal diffractive structure function $F_{L}^{D}$ is the first of its kind and is presented by the H1 Collaboration using the latest HERA data dedicated especially for the $F_{L}$ and $F_{L}^{D}$ analyses. This textbook measurement could only be carried out using data at different centre of mass energies. The measured $F_{L}^{D}$ fully supports the concept of QCD factorisation in diffraction and independently cross checks the gluon parton densities. It is an important test of our understanding of the nature of diffractive processes and it complements the diffractive physics programme at $\mathrm{H} 1$.

\section{References}

[1] Slides: http: //indico. cern. ch/materialDisplay py?contribId=111\&sessionId=18\&materialId=slides\&conf Id=53294

[2] K. Golec-Biernat and A. Luszczak, Diffractive Parton Distributions from Fits with Higher Twist, arXiv:0807.0724 [hep-ph] (2008).

[3] A. Glazov, these proceedings.

[4] H1 Collab., F.D. Aaron et al., Published in Phys. Lett. B665 (2008) 139-146.

[5] P.R. Newman, Prospects for $F_{L}^{D}$ Measurements at HERA-II, hep-ex/0511047 (2005).

[6] H1 Collab., A. Aktas et al., Eur. Phys. J. C48 (2006) 715-748. 